\title{
Point of (Re)View
}

\section{Subashree Krishnaswamy}

\begin{abstract}
This paper focuses on why translations should be reviewed differently from original writings. What are the different ways in which a translation is usually reviewed? Is there really a best way to read a translation? Is it necessary for a reviewer to know the source language? Why should the reviewer be translation-sensitive? The paper draws on experiences of the author as an editor of a review magazine $3 / 4$ 'Indian Review of Books' 3/4 which regularly reviewed literatures in translation.
\end{abstract}

Should a translation be read differently from an original piece of writing? I am always nettled that such a question should even be entertained, even though as editor of the review magazine Indian Review of Books (IRB), I was asked precisely this any number of times. After all, isn't it evidently clear that the reader is not encountering the author's work, only the translator's rendering of it? As the well-known translator and critic Lawrence Venuti put it succinctly in his article, 'How to Read a Translation': 'A translation has to be read differently from an original composition precisely because it is not an original...' (Venuti 2009) That the work comes to us 'filtered' through the 'translator's lens' can never be forgotten or ignored.

A number of scholars have noted the various ways in which reviewers read translations, and I can only reaffirm what they have said. (I first came across the terms 'translation-blind', 'translationaware' and 'translation-sensitive' in Anne Milano Appel's excellent essay on reviewing in the ATA Chronicle and I am taking the liberty of using them in this essay since they so aptly describe the situation in India as well). I will be only touching upon translations into English because that is what I have worked closely with for a number of years.

Translation Today Vol. 5 No. 1 \& 22008 @ CIIL 2008 
First, there are those reviewers who are 'translation-blind,' who disregard - deliberately or otherwise - the fact that the book under review is a translation, lending credence to the popular notion of the translator's invisibility. Are these reviewers under the impression that they are flattering the authors, or do they unquestionably believe that the translation is perfect? The book under review, they affirm, is after all in English, and must be treated like any other of its genre in the language; when questioned closely, they disclose that it is the English version they are bothered about, and in any case they do not know the original language and much less do they care about the origins. They are therefore merely interested in advising the reader and not bothered about the process at all. Often, publishers themselves are guilty, unwilling as they are to advertise that the books are translations the translator's name is usually printed in an obscure corner. Perhaps this in-built block attached to translations has much to do with marketability.

The second kind of translators is 'translation-aware': they usually pay token respect to the fact that the book is a translation. But they are also the ones who usually summarise the book, borrow happily from the blurb/introduction without acknowledging, and punctuate their writing with 'graceful' or 'excellent,' without ever furnishing examples of sentences that demonstrate the worthiness of such epithets. Among this category are also the ones who want to say something, but don't know what to say, really. Perhaps the reputation of the author intimidates such reviewers, and therefore when confronted with staccato or flat, functional prose, they make the translators the scapegoat, clearly forgetting that no translator can take it upon himself/ herself to fix the prose. This is not to say that pedestrian writing should be condoned, but a few examples would certainly be in order.

We all know the nitpickers of course, the ones who are familiar with the original, but usually review only to pick holes. They are the ones given to sweeping statements, which are however left unfailingly unexplained. 'This does not do justice to the original' is a favourite refrain. Surely all translators work on the premise that something will 
inevitably be lost in translation, especially if the target and original languages straddle different cultures. So what is the purpose in saying that one ought to read it in the original? This is not to say that bad translations should never be slammed, but if a book is that bad, does it merit a review at all in the first place? Of course if an unworthy book has earned unwarranted publicity and notice, it might be useful to enumerate the flaws.

Nitpickers also review to triumphantly show off their knowledge and home in on the odd error or two; it could be something as inconsequential as 'soft hair' on the arm mistakenly translated as 'soft skin.' Yes, this is a mistake that ought to be pointed out, but to devote entire paragraphs to something merely descriptive, which doesn't contribute in any big way to the narrative, is mere quibbling or bragging. It was an oversight on the part of the translator, but surely just three or four tiny errors in four hundred odd pages of otherwise competently translated dense material are excusable. A vigilant translator would certainly correct them in the next edition, but to judge his/her competency on such trivial matters is petty and meaningless.

A few words about the question of invisibility are in order. All of us, translators included, are quite happy to hear praise such as 'it reads so well that one forgets it is a translation.' In fact, it is always presumed that the better a translation reads, the less we think about the translator and the more invisible the translator becomes. Is this really fair? To quote Lawrence Venuti once again, 'We typically become aware of a translation only when we run across a bump on its surface....' (Venuti 2009) If the bump is occasioned by the visibility of the translator's hand-not error in usage or a confused meaning that may seem intentionally comical—which allows the cadences of the original language and culture to be heard, then it is a good translation. Nowadays translators do not want the original language to be tamed by English; rather they strive to expose readers to the uniqueness of the other language. And rightly so. The bumps in our books usually take the form of kinship terms, forms of address, expressions, proverbs, idioms, and dialogues which use English as it is spoken today with all the regional variations. 
Which is where translation-sensitive reviewers score. They are reviewers who never lose sight of the fact that the book is a translation and view the translator as a special kind of writer, possessing not an originality that competes with the author's, but rather an art which uses the stylistic devices that tap into the literary resources of both the languages. A translation communicates not merely a text but the translator's interpretation of it. Sensitive reviewers, often familiar with the target and source texts, make judicious comparisons and also manage to talk about the issues that informed the work and culture of the original work.

Does that mean that every reviewer should know the original? That is the ideal situation, of course, but not entirely feasible. Besides, translations are meant for readers who don't know the original language. We at IRB carried out an interesting experiment. We commissioned two reviews of the same book, one by a reviewer familiar with the original and one by a practising translator in another Indian language. The one who didn't know the original was delighted, even grateful, for the competent translation, which introduced him to a stalwart who would otherwise have remained unknown to him. But the one who knew the original was clearly unhappy with certain choices and elaborated upon them.

How does one judge then? We realized that there was no one perfect way to review translation. Every reviewer brings a different set of abilities to the task. But good reviewers always keep certain things in mind-they never ignore the fact that it is a translation they are reviewing; they are clear that what they are reading is not merely the author's writing, but a translator's rendering of it; and they are also aware that they are looking at a work through the lens of a translator. They will unhesitatingly use expressions such as 'as rendered by the translator,' affirming that a translation is an independent text and it is only courtesy the translator that a reviewer can access the original.

Was IRB an exemplary magazine then? Sadly not, and the reasons were many. Like most things literary, we were always strapped 
for cash. This meant that we sometimes had to accept mediocre reviews even after sending them back for rewriting since we owed it to the publishers who strongly believe that any publicity was better than no publicity at all. Reviewers accepted books with alacrity, but remorselessly reneged on deadlines. Had we a corpus, we would have set aside a tidy amount to take care of 'kill fee.' Receiving two review copies from publishers wouldn't have hurt either since it was often only in hindsight (when we read the book) that we realized that some reviews were clearly unjust. Which is why heated, lively letters from readers sustain a good review magazine, and of that I'm glad to say we received in plenty.

It is high time seasoned reviewers remove their blinkers. A word of caution however: a translated text cannot be seen with the naked eye, it needs the lens of an able translator.

\section{Reference}

Venuti, Lawrence (2009) 'How to Read a Translation' http://www.wordswithoutborders.org/?lab=HowTo 